\title{
To bee, or not to bee? Honey bees, Boolean logic, bits and information
}

\section{Fiona Edwards Murphy}

Electrical and Electronic Engineering (EEE), School of Biological, Earth and Environmental Sciences (BEES), and Environmental Research Institute (ERI), UCC

Human beings have fabricated the illusion that in the 21st century they have the technological prowess to be independent of nature. Bees underline the reality that we are more, not less, dependent on nature's services in a world of close to 7 billion people. (Achim Steiner, Executive Director UN Environment Programme)

\section{The importance of Honey Bees}

What would happen if the honey bee disappeared from the face of the earth? Honey bees do not just produce the honey and wax we use every day; they serve the vitally important function of pollination, in which pollen is moved from one plant to another for fertilisation. 70 of the 100 crop species which provide $90 \%$ of food worldwide are pollinated by bees. The value of honey bee pollination to the global economy ( $€ 153$ billion annually) now vastly outstrips the value of honey and beeswax combined. As the human population worldwide continues to grow, the demand for pollinator dependant crops will continue to increase proportionally. It is safe to say that the disappearance of the honey bee would pose a global crisis for humanity.

In the past few years there has been a renewed buzz about the plight of the honey bee. A range of honey bee pests and diseases have recently become increasingly widespread, with populations in decline across Europe, America, and Asia. The rapid mortality of a colony is usually described as Colony Collapse Disorder, or CCD. This is a phenomenon where the adult population of a hive disappear or die. A range of possible causes for the current population failure have been proposed including pesticides, fungicides, selective breeding, and antibiotics, leading to an EU wide ban on pesticides. Despite this heightened research effort, scientists have not been able to identify a conclusive source of this decline in population.

Maximising the productivity of the existing honey bee population, as well as providing opportunities for growth in the face of spreading worldwide pests and diseases have now become hot topics for research around the world. My project aims to use "Wireless Sensor Network" (known as WSN) technology to remotely monitor the condition of beehives, in order to inform the beekeeper of the colony's status. In relation to diseases, this technology 
can provide concrete evidence of the changes in the hive. If early evidence of disease can be detected, beekeepers can intervene earlier and thus improve the yield of their hives by lowering bee mortality.

Many great minds throughout history have been inspired by honey bees, from philosophers (Aristotle was one of the first to observe communication between honey bees, now known as the famous "waggle dance"), to scientists (Charles Darwin noted in On the Origin of Species that honey bees are "absolutely perfect in economising labour and wax."), to writers (William Shakespeare compares a human monarchy with a beehive in Henry $V$, "Creatures that by a rule in nature teach/ The act of order to a peopled kingdom."). This inspired the title of the project: a pun on the famous Shakespearean quote, "to be, or not to be?".

\section{Honey bees in Cork and Ireland}

Ireland, and in particular Cork County, has a strong history relating to honey bees, with some of the oldest surviving documents describing the abundance of honey in ancient Ireland. A historic tradition of beekeeping is shown clearly in "An Bechbretha", or "beejudgements", Irish Brehon laws (7th Century) relating to beekeeping, covering topics such as ownership of swarms, theft of bee hives, and neighbours' entitlements to honey. The patron saint of beekeeping is Saint Gobnait, who is said to have cultivated bees in Ballyvourney, Co. Cork, where a shrine to her remains today (Fig. 1). One story tells of how she loosed her bees upon invaders to prevent them from stealing cattle. A stained glass window in the Honan Chapel in University College Cork depicts her surrounded by honey bees (Fig. 1). This strong history, as well as the continuing popularity of beekeeping in Ireland, makes Ireland an ideal location for tackling the global issues surrounding honey bees.

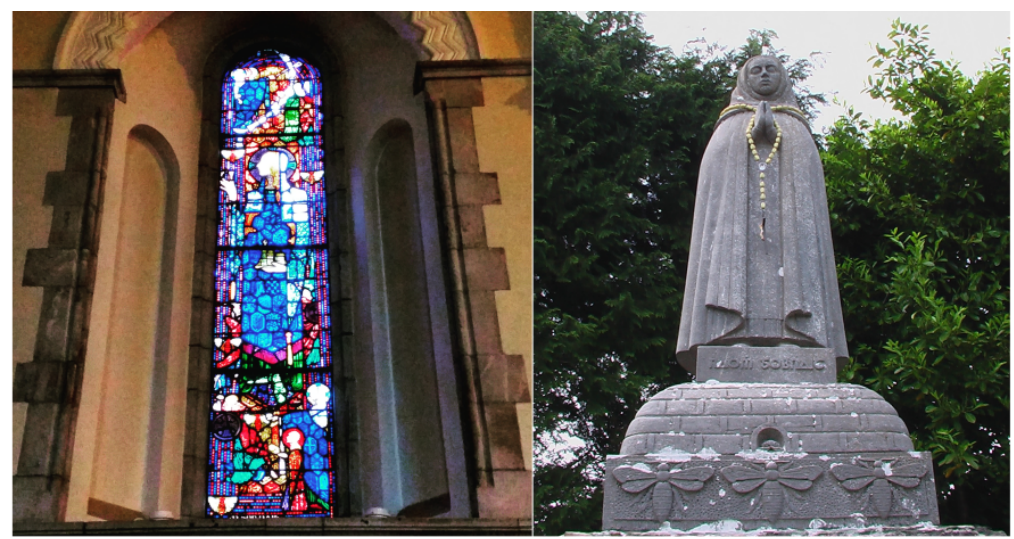

Figure 1: Saint Gobnait depicted in the Honan Chapel and at a shrine in Ballvvourney. Source: John Staats, Link: https://commons.wikimedia.org/wiki/ File:Saint_Gobnait.jpg licensed under CC BY 3.0 http://creativecommons.org/ licenses/by/3.0/ 


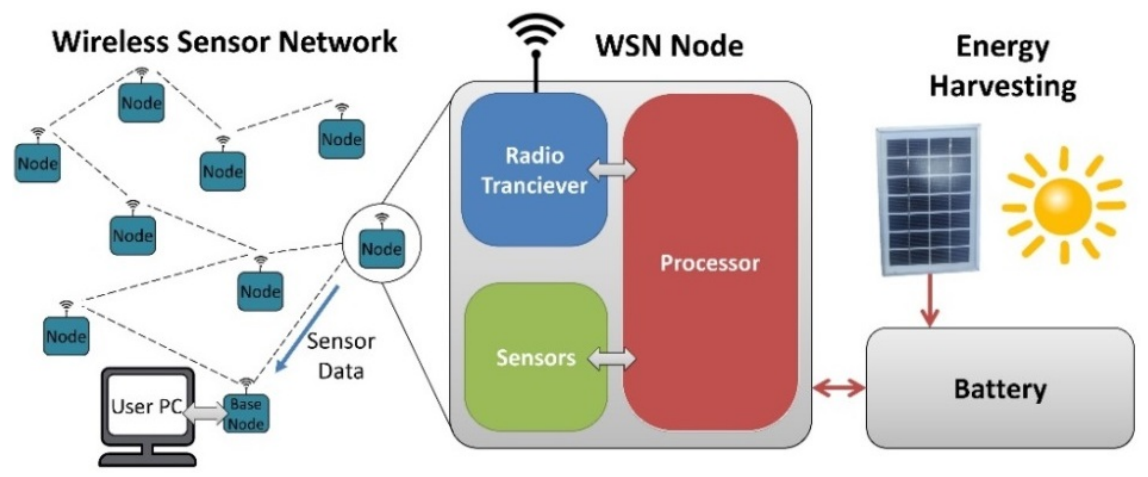

Figure 2: WSN operation. Source: Author

\section{Boolean logic and bits}

My project proposes to create a system for monitoring beehives remotely using wireless sensor networks, together with cloud storage and signal processing.

\section{What are Wireless Sensor Networks?}

Wireless Sensor Networks (WSN) is a unique technology which allows the user to gather data about physical or environmental parameters, often over a wide area, using a series of physically separate "nodes". Each node is made up of one or more sensors (as the application requires); a battery or power supply; and a low power processor, which controls the node's activity. The nodes are also enabled with wireless communication capabilities, allowing them to pass collected data to each other. This allows them to "funnel" all of the data to a single node for collection and analysis.

The operation and architecture of a WSN is described in Fig. 2. Applications of WSN include smart homes, security, video surveillance, industrial automation, and e-health. WSN is a vital technology for realising the emerging concept of the "Internet of Things" (IoT), a scenario where everyday objects are connected to the internet, allowing them to work together, tracking user requirements, improving accuracy, and reducing waste.

\section{Boolean logic}

WSN, like most modern communication technologies, utilises Boolean algebra at its very core. George Boole invented Boolean algebra and symbolic logic and is described as the 'father of the information age'. He was appointed as the first Professor of Mathematics at UCC in 1849. In my project information from the bees is stored as Boolean "bits". In fact the title of this article can be described technically to engineers and computer scientists entirely as a Boolean equation: (2B)OR!(2B)=TRUE. 


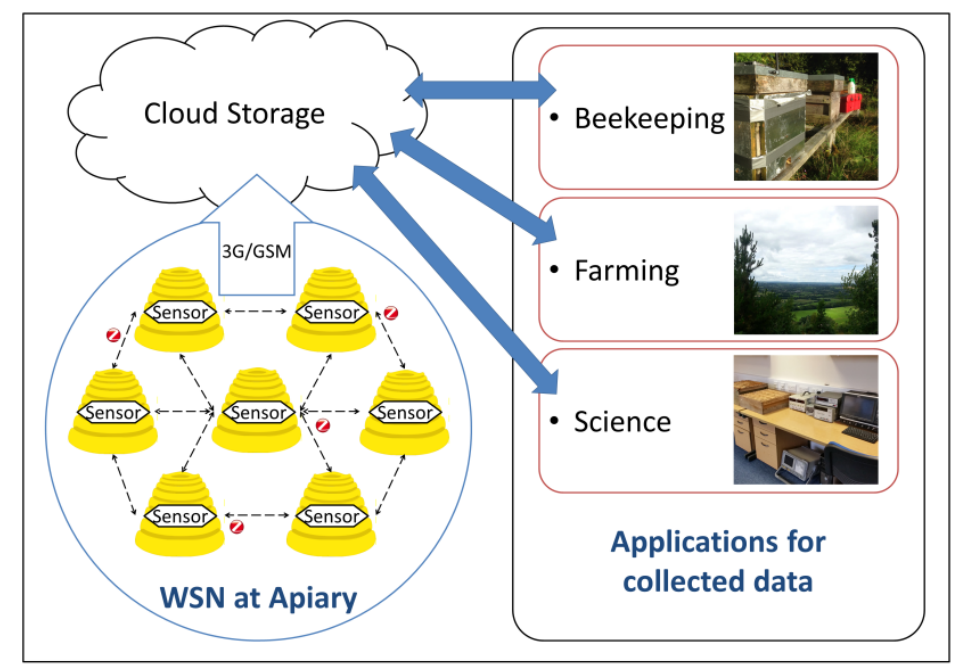

Figure 3: From the beehive to the cloud. Source: Author

\section{The Smart Beehive project}

My project places a series of wireless sensing devices, or "nodes", within live beehives. These nodes gather data on several hive parameters which are important to the beekeeper for managing pests, disease, swarming and production. These parameters include $\mathrm{CO}_{2}$ (carbon dioxide), $\mathrm{O}_{2}$ (oxygen), pollutant gas levels, temperature, humidity, beehive weight, movement, sound levels, and bee imagery. These data are uploaded from the WSN to Cloud storage, where they can be processed and accessed by the beekeeper through a mobile device/PC. A diagram of how the envisioned system operates is shown in Fig. 3.

Several important features for the hive system were identified:

$\square$ Non-invasive, minimum impact on hive and honey bee colony. This is necessary to avoid a negative effect on colony health, production and size.

$\square$ Does not disturb/prevent typical beekeeper activities. The system must not impede regular inspection of the hive, and the beekeeper must have quick access to the hive in an emergency.

$\square$ Robust and resistant to hive conditions. The beehive is a hostile environment for electronics/batteries as it is hot and humid. Honey bees also cover anything within the hive for extended periods in propolis and/or wax, which could impact sensor readings.

Low energy operation \& energy harvesting. This allows extended deployments of the system.

Suitable for remote deployment. Beehives and apiaries are often placed in isolated locations, and the design of the system needs to reflect this property.

The final smart hive design can be seen above in Fig. 4, the nodes are placed in the "lid" 

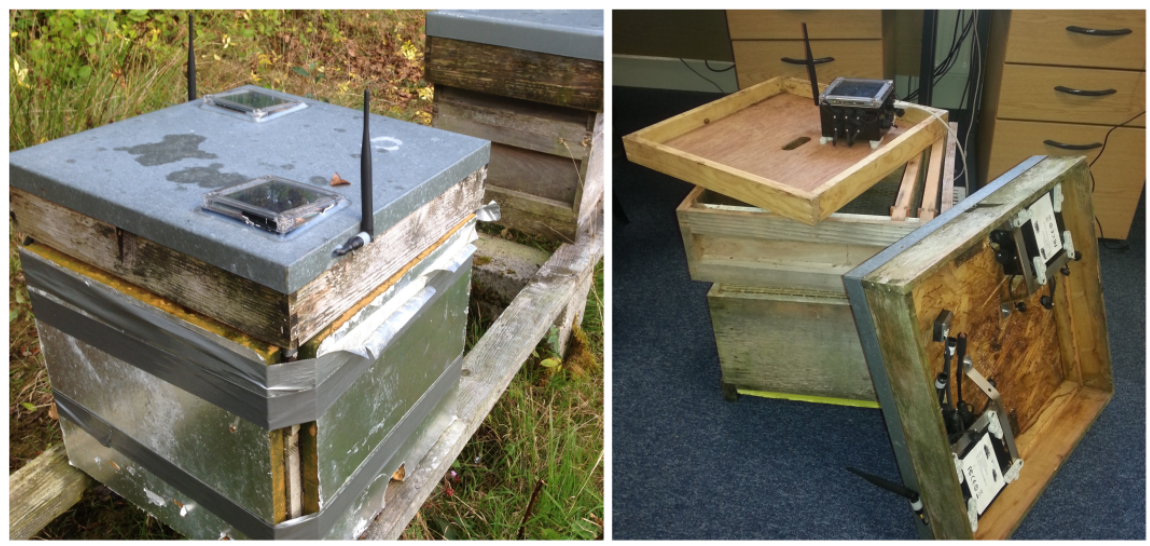

Figure 4: Smart hive prototype and layout. Source: Author

of the hive so that they can be removed by the beekeeper when they want to inspect their bees in person. Placing the sensors inside the lid also allows them to effectively sample the airflow of the hive without being covered in wax, as the bees do not often enter the lid space. The data collected by the sensors are sent to the beekeeper, allowing them to view the conditions within the hive when they are unable to visit, such as during the winter, night time, or when the hive is in an extremely remote location. The sensor network uses a $3 \mathrm{G}$ radio (the same as in a modern smartphone) to send data to our storage servers and the beekeeper. This radio uses the existing mobile network to send data, without needing services such as a landline internet connection to every hive location. The prototype system shown was deployed on a live beehive during 2014 at an apiary near Banteer, Co. Cork, Ireland. Weather data for the duration of the deployment was obtained from Met Éireann to provide an extra dimension for analysis. The results of this deployment were vital in improving future versions of the system and developing automatic hive analysis systems described below.

\section{Self-Sustainable operation}

Just like every other electronic device, the smart hive requires power to operate. Obviously plugging the hive in is not an option when the hive is on the side of a mountain or in the middle of a forest. A battery would be able to power the hive for a while but would eventually run out. The beekeeper would then need to travel to every hive and replace the battery which defeats the purpose of remote data collection! To avoid this, a state known as "self-sustainable operation" needs to be achieved. This is where each node has a rechargeable battery together with "energy harvesting" devices, such as a solar panel or a wind turbine, to recharge it. Self-sustainable operation is achieved when the battery is regularly charged up by at least as much energy as the node uses in that period, meaning that the device effectively never runs out of power.

Solar panels have been successfully used in the initial deployment of the smart hive. This will be continued in the future deployments, together with a small wind turbine which 


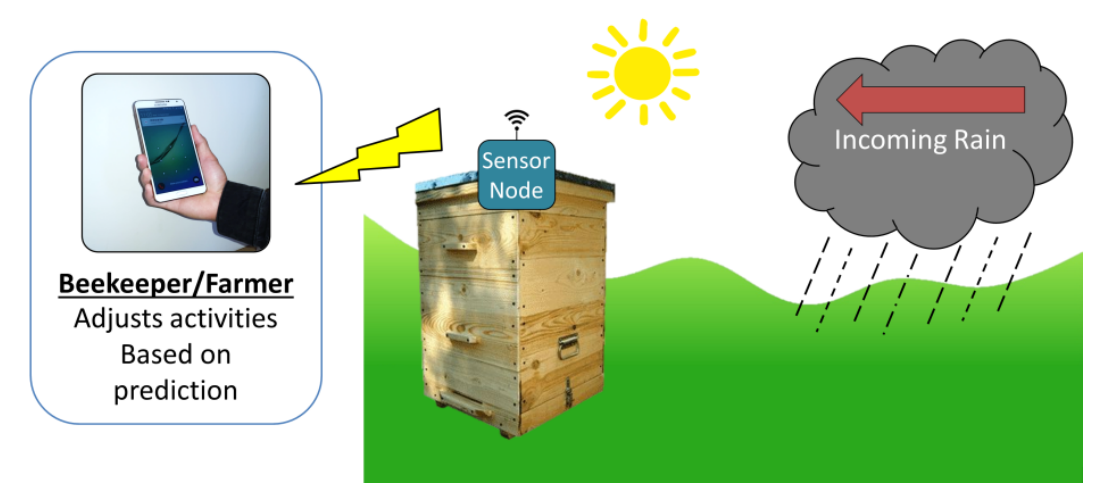

Figure 5: Predicting Irish weather, an "NBee Complete" challenge. Source: Author

will provide more energy during winter deployments. This is important because opening the hive to replace a battery, allowing cold air in to the hive, could be detrimental to the colony. More energy will also allow more data to be collected and stored, as each "bit" of information requires a certain amount of energy to collect, store, and send; and more information $=$ more bits $=$ more energy.

\section{Information - The future of beekeeping}

The main objective of this work from a beekeeping perspective is to provide an effective tool for high accuracy beehive management. A beekeeper that is well-informed about the status of their hives can apply their time and resources in a much more effective manner. In this way the smart hive can make beekeeping a more lucrative activity in the future. To ensure the smart hive has maximum effect, it is important that it can not only provide large amounts of raw data, but can extract information about the in hive activity and bring this information to the keeper's attention.

One key engineering contribution of my work is in the area of machine learning, which is the development of algorithms which can extract information, make decisions, and make predictions based on data without the need for human intervention. Initially I have developed two machine learning algorithms, one of which can automatically describe the status of the hive (healthy, producing honey, hibernating, poorly ventilated, unwell/diminished population, or dead), based on this description the smart hive can select to send the beekeeper a text alert that the hive needs immediate attention. The second algorithm in development can accurately predict if there will be rain in the area local to the beehive in the next few hours, which has the potential to be a very useful tool for farmers as well as beekeepers.

I would like to thank my supervisors, Dr. Emanuel Popovici and Dr. Pádraig Whelan for their encouragement and support. I would also like to gratefully acknowledge funding provided by the Irish Research Council under their Government of Ireland Postgraduate Scholarship 2014 and UCC's Strategic Research Fund. This project was awarded 1st prize in the IEEE /IBM Smarter Planet Challenge 2014. 